SLAC-PUB- 8175

May 99

Measurements of $Z^{0}$ Electroweak Couplings at SLD ${ }^{1}$

\title{
Giampiero Mancinelli
}

Rutgers University, New Brunswick, New Jersey 08903

Representing The SLD Collaboration ${ }^{\dagger}$

Stanford Linear Accelerator Center

Stanford University, Stanford, CA 94309

\begin{abstract}
In this paper we report a summary of the results of several electroweak measurements performed by the SLD experiment at the Stanford Linear Collider (SLC). Most of these results are preliminary and are based, unless otherwise indicated, on the full 1993-1998 dataset of 560,000 hadronic $Z^{0}$ decays, produced with an average electron beam polarization of $73 \%$.

Invited talk presented at the 13th Rencontres de Physique de la Vallee d'Aoste: Results and Perspectives in Particle Physics La Thuile, Italy 28 February - 6 March 1999
\end{abstract}

\footnotetext{
${ }^{1}$ This work was supported by Department of Energy contracts: DE-FG02-91ER40676 (BU), DE-FG0392ER40701 (CIT), DE-FG03-91ER40618 (UCSB), DE-FG03-92ER40689 (UCSC), DE-FG03-93ER40788 (CSU), DE-FG02-91ER40672 (Colorado), DE-FG02-91ER40677 (Illinois), DE-AC03-76SF00098 (LBL), DE-FG02-92ER40715 (Massachusetts), DE-AC02-76ER03069 (MIT), DE-FG06-85ER40224 (Oregon), DEAC03-76SF00515 (SLAC), DE-FG05-91ER40627 (Tennessee), DE-AC02-76ER00881 (Wisconsin), DE-FG0292ER40704 (Yale); National Science Foundation grants: PHY-91-13428 (UCSC), PHY-89-21320 (Columbia), PHY-92-04239 (Cincinnati), PHY-95-10439 (Rutgers), PHY-88-19316 (Vanderbilt), PHY-92-03212 (Washington); the UK Particle Physics and Astronomy Research Council (Brunel and RAL); the Istituto Nazionale di Fisica Nucleare of Italy (Bologna, Ferrara, Frascati, Pisa, Padova, Perugia); and the Japan-US Cooperative Research Project on High Energy Physics (Nagoya, Tohoku).
} 


\section{Theoretical Overview}

The $S U(2)_{L} \times U(1)$ Standard Model (SM) has the following Feynman rule for neutral current (NC) fermion $-Z^{0}$ boson interaction:

$$
\frac{-i}{\sqrt{2}}\left(\frac{G_{F} M_{Z}^{2}}{\sqrt{2}}\right) \bar{f} \gamma^{\lambda}\left(g_{V}-g_{A} \gamma_{5}\right) f Z_{\lambda}
$$

where $G_{F}$ is the Fermi constant, $M_{Z}$ the $Z^{0}$ mass and $g_{V}$ and $g_{A}$ the vector and axial-vector fermion couplings:

$$
g_{V}=\left(g_{L}+g_{R}\right)=I_{L}^{3}-2 Q \sin ^{2} \theta_{W} \quad g_{A}=\left(g_{L}-g_{R}\right)=I_{L}^{3} .
$$

$I_{L}^{3}$ is the third component of the fermion weak isospin, Q the fermion electric charge and $\theta_{W}$ the Weinberg angle.

The observables at the $Z^{0}$ pole are the following combinations of these couplings: $A_{f}$, which represents the extent of the parity violation at the $Z f \bar{f}$ vertex:

$$
A_{f}=\frac{2 g_{V} g_{A}}{g_{V}^{2}+g_{A}^{2}}=\frac{g_{L}^{2}-g_{R}^{2}}{g_{L}^{2}+g_{R}^{2}},
$$

and $R_{f}$, the fraction of $Z^{0} \rightarrow f \bar{f}$ events in hadronic $Z^{0}$ decays:

$$
R_{f}=\frac{\Gamma\left(Z^{0} \rightarrow f \bar{f}\right)}{\Gamma\left(Z^{0} \rightarrow \text { hadrons }\right)} \propto g_{L}^{2}+g_{R}^{2} .
$$

The precise measurements of these quantities should ultimately verify whether or not the couplings for all generations and weak isospin states are given by the theory isospin assignments and a universal value of $\sin ^{2} \theta_{W}$.

The differential cross section for $e^{+} e^{-} \rightarrow Z^{0} \rightarrow f \bar{f}$ and longitudinally polarized electrons is given by:

$$
\frac{d \sigma}{d z} \propto\left(1-P_{e} A_{e}\right)\left(1+z^{2}\right)+2 z\left(A_{e}-P_{e}\right) A_{f},
$$

where $z$ is the cosine of the polar angle of the outgoing fermion $f$ with respect to the incident electron, $P_{e}$ the electron beam longitudinal polarization, and $A_{e}$ and $A_{f}$ the asymmetry parameters for the initial and final state fermions, respectively.

The presence of the parity violation parameters introduces a forward-backward asymmetry, which is, for unpolarized beams:

$$
A_{F B}^{f}(z) \equiv \frac{\sigma_{f}(z)-\sigma_{f}(-z)}{\sigma_{f}(z)+\sigma_{f}(-z)}=A_{e} A_{f} \frac{2|z|}{1-z^{2}} .
$$

In the presence of $e^{-}$beam polarization, as at the SLC, it is possible to construct the left-right forward-backward asymmetry

$$
\begin{aligned}
\tilde{A}_{F B}^{f}(z) & \equiv \frac{\left[\sigma_{L}^{f}(z)-\sigma_{L}^{f}(-z)\right]-\left[\sigma_{R}^{f}(z)-\sigma_{R}^{f}(-z)\right]}{\left[\sigma_{L}^{f}(z)+\sigma_{L}^{f}(-z)\right]+\left[\sigma_{R}^{f}(z)+\sigma_{R}^{f}(-z)\right]} \\
& =\left|P_{e}\right| A_{f} \frac{2 z}{1+z^{2}},
\end{aligned}
$$


for which the dependence on the initial state coupling parameter $A_{e}$ disappears, allowing a direct measurement of the final state coupling parameters $A_{f}$. Thus electron beam polarization permits a unique measurement of $A_{f}$, independent of that inferred from the unpolarized forward-backward asymmetry[1] which measures the combination $A_{e} A_{f}$, and gives a statistical advantage of $\left(P_{e} / A_{e}\right)^{2}$ which is roughly equivalent to a factor 25 at the SLC.

The left-right cross section asymmetry is defined as:

$$
A_{L R}^{\text {meas }} \equiv \frac{\sigma_{L}-\sigma_{R}}{\sigma_{L}+\sigma_{R}}=P_{e} A_{e}
$$

It yields a very precise direct measurement of the initial state $Z e e$ coupling and gives a very sensitive measurement of the weak mixing angle since $\delta A_{e} \sim 8 \delta\left(\sin ^{2} \theta_{W}\right)$. Measuring and comparing the asymmetry parameters $A_{f}$ for the different lepton types also provides a direct test of lepton universality, which is assumed in the SM.

Precise measurements of the asymmetry parameters $A_{f}$ and the partial widths $R_{f}$ can probe the effect of radiative corrections to the $Z^{0}$ propagator or to the the $Z f \bar{f}$ vertex. Since the radiative corrections depend on the top and Higgs masses, precise electroweak measurements can also measure or constrain these quantities.

In particular, especially interesting is the measurement of the $Z^{0}$ to $b$ quark couplings for several reasons. First, the quantity $A_{b}$ is largely independent of propagator effects that modify the effective weak mixing angle, hence it is complementary to other electroweak measurements performed at the $Z^{0}$ pole. Second, physics beyond the SM may couple more strongly to third generation fermions, producing larger deviations in $b$ couplings than in other quark couplings. Finally, since for $b$ quarks $g_{L}^{2} \simeq 30 g_{R}^{2}$, the observable $R_{b}$ has large sensitivity to possible deviations from the predicted left-handed coupling between the $Z^{0}$ and $b$-quarks and is complementary to the measurement of $A_{b}$, which, instead, has greater sensitivity to right-handed components.

\section{SLD/SLC Unique Features}

The recent (1997-98) performance of the SLC has been excellent, with peak luminosities of $3 \times 10^{30} \mathrm{~cm}^{-2} \mathrm{~s}^{-1}\left(20,000 Z^{0}\right.$ decays/week), nearly a factor of three improvement compared to the best previous results. The total number of $Z^{0}$ hadronic decays collected during the running period 1993-98 has raised to about 560,000.

A detailed description of the SLD detector can be found elsewhere[2]. Here are listed some of the unique features which allow the SLD experiment to perform many competitive electroweak and heavy flavor physics measurements:

- Highly longitudinally polarized (average $~ 73 \%$ ) electron beams.

- A small and stable beam spot $(1.5 \times 0.8 \times 700 \mu \mathrm{m})$ and a high precision 3D CCD-based pixel vertex detector (Interaction point (IP) determined to $6 \times 6 \times 25 \mu \mathrm{m}$ with an impact parameter resolution of $11 \times 23 \mu m(r \phi \times r z)$ for high momentum tracks).

- Good particle identification, using a Čerenkov Ring Imaging Detector (CRID). 


\section{Lepton Coupling Asymmetry Meaurements}

\subsection{Left-Right Cross Section Asymmetry $\left(A_{L R}\right)$}

$A_{L R}$ is an inclusive measurement that depends only on the initial state electron couplings and the magnitude of the electron beam polarization. No efficiency or acceptance effects enter and no significant final state identification is required, consequently practically all of the data can be used and this contributes to the high statistical precision of this measurement. Nevertheless, due to the high precision of the polarization measurement, the results is still statistically limited (systematic error $\sim .75 \%$, statistical error $\sim 1.3 \%$ ). Most recently, a dedicated experiment using the End Station A fixed target polarimeter confirmed that accidental $e^{+}$polarization is consistent with zero $(-0.02 \pm 0.07 \%)$.

The $A_{L R}$ measurement uses all hadronic events and is effectively a counting experiment. In fact, with equal luminosity for right- and left-handed events, we can replace the cross sections in equation 8 with the number of events $N_{L}$ and $N_{R}$, so that $A_{e}=\frac{1}{P_{e}} \frac{N_{L}-N_{R}}{N_{L}+N_{R}}$. Additional corrections must be applied due to direct channel $\gamma$-exchange and from $\gamma Z^{0}$ interference. After applying these corrections, which are well known and of the order of $\sim 2.5 \%$, we measure $\sin ^{2} \theta_{W}=0.23101 \pm 0.00031$.

The quantity $A_{L R}$ can be measured only in the presence of polarized beams and provides by far the most precise determination of $\sin ^{2} \theta_{W}$ presently available, without need to assume lepton universality.

\section{2 $A_{\text {lepton }}$ Measurements from $\tilde{A}_{F B}$}

$A_{e}, A_{\mu}$ and $A_{\tau}$ are measured at SLD from leptonic decays of the $Z^{0}$ boson via the corresponding left-right forward-backward asymmetries for each lepton type. A likelihood fit is used to simultaneously measure $A_{e}$ and $A_{l}$ (where $l=e, \mu, \tau$ ). SLD's $A_{\mu}$ constitutes the world's best measurement, while $A_{\tau}$ is best measured at LEP I, by combining the results for all four experiments. The SLD $A_{\tau}$ result is statistically comparable to one LEP I experiment. The SLD results are consistent with lepton universality (see Table 1). Assuming lepton universality, we can combine these results with that from $A_{L R}$ for a measurement of $\sin ^{2} \theta_{W}$ of $0.23109 \pm 0.00029[3,4]$.

\section{$4 \quad R_{b}$ and $R_{c}$ Measurements}

The measurements of $R_{b}[5]$ and $R_{c}[6]$ heavily exploit the capabilities of the SLD vertexing via a topological vertex algorithm[7], which has proven to be efficient and robust. After a standard hadronic decay selection, each event is divided into two hemispheres where secondary (and tertiary) vertices are found. Secondary vertices are found in $65 \%$ of the $b$ hemispheres and in $20 \%$ of the $c$ hemispheres. The vertex invariant mass is calculated and corrected for missing transverse momentum. Hemispheres are tagged as containing a $b$ quark if their corrected vertex mass is greater than $2 \mathrm{GeV}$ and the secondary vertex is at least 5 standard deviations away from the primary vertex. The hemisphere purity for $b$ events is $\sim 98 \%$ with an efficiency of $\sim 50 \%$. Events are selected and tagged as $b \bar{b}$ if at least one of their hemispheres satisfies the previous conditions.

Similarly, an hemisphere is tagged as $c \bar{c}$ if the following conditions are satisfied: 

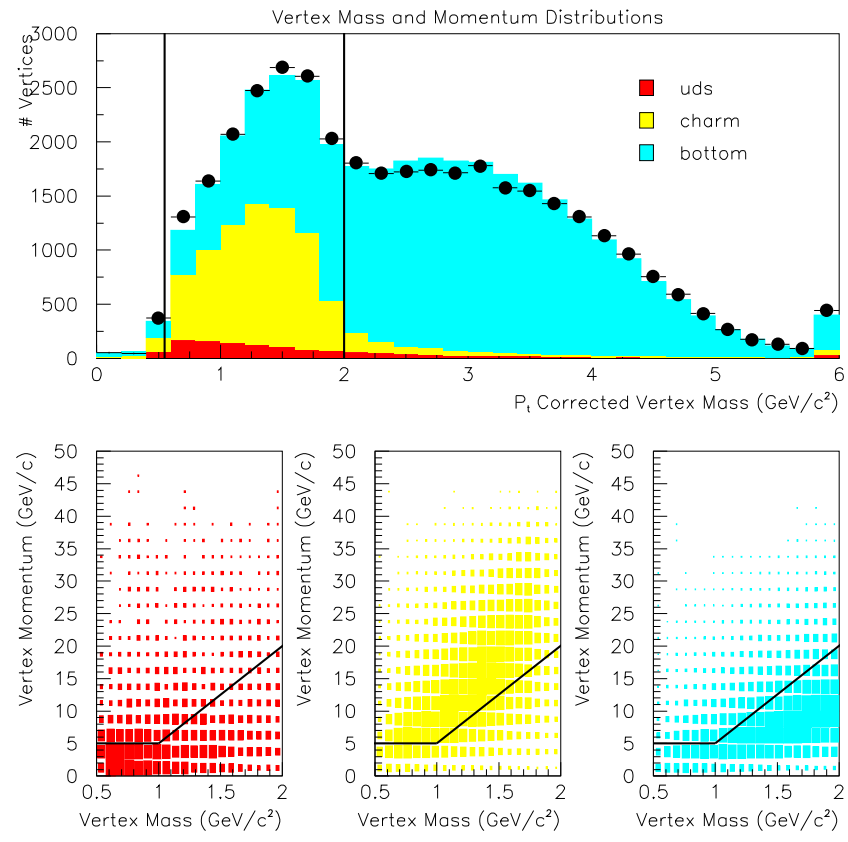

Figure 1: Top: distribution of the vertex mass hemisphere for data and Monte Carlo. Bottom: vertex mass vs vertex momentum for $u d s$ (left), charm (middle) and $b$ (right) events.

- At least one track present with 3D impact parameter more than 3 standard deviations from the primary vertex.

- $0.55<M_{v t x}<2 \mathrm{GeV}$.

- $P_{v t x}>5 \mathrm{GeV}$.

- $P_{v t x}>15 M_{v t x}-10 \mathrm{GeV}$.

The last cut uses the fact that $b$ events that populates the low mass region, do so because they are missing some tracks and, consequently, have a lower momentum for the same vertex mass (see fig 1). The resultant hemiphere purity is $\sim 70 \%$ with $\sim 16 \%$ efficiency.

The charm and bottom tagging efficiencies are self-calibrated, using the single, double and mixed tag rates, directly from the experimental data. The Monte Carlo (MC) is used as input for the $c$ and $u d s$ efficiencies in the $b$ tag region. For this reason it is very important to have high purity in the sample of events tagged as $b$, in order to reduce the systematic uncertainties due to modelling of the charm production and decay in the MC. Hemisphere correlations are also derived from the MC.

We measure: $R_{b}=0.2159 \pm 0.0014$ (stat) \pm 0.0014 (syst) and $R_{c}=0.1685 \pm 0.0047$ (stat) \pm 0.0043(syst). $150,000 Z^{0}$ hadronic decays from the last part of the 1998 run have not yet been included in the $R_{b}$ result.

\section{Quark Coupling Asymmetry Measurements $\left(A_{q}\right)$}

SLD provides several methods to measure the quantites $A_{q}$, which are largely independent. Their statistical and systematic correlations are taken into account in the combinations of their results. All these measurements construct the left-right forward-backward asymmetries 
defined previously and use, in different ways, the secondary vertex information. Three different techniques are employed to measure the parity violating parameter $A_{b}$. They use respectively the jet charge, the kaon charge and the lepton charge to tag the $b$ and the $\bar{b}$ hemispheres. Four methods are instead used to determine $A_{c}$. To determine the $c$ hemisphere they use the kaon or vertex charge, the lepton charge, the soft pion charge or $D^{(*)}$ meson decays reconstructed exclusively. Finally, SLD is able to produce a very good measurement of $A_{s}$, using identified kaons and $\Lambda$ particles.

\section{$5.1 \quad A_{b}$ with a Kaon Tag}

This measurement[8] exploits the decay chain $b \rightarrow c \rightarrow s$ to tag the initial $b$ quark sign. It heavily relies on the good kaon identification from the CRID detector and the excellent separation between kaons coming from the IP and those from the secondary vertex. The error is at this moment dominated by the uncertainty in the $K^{+} / K^{-}$production ratio in $B$ meson decays, but it will likely improve soon employing a self-calibrated method. The $A_{b}$ value from this measurement (derived only from the 1994-95 data sample) is $0.855 \pm$ 0.088 (stat) \pm 0.102 (syst).

\section{2 $A_{b}$ with a Momentum Weighted Track Charge}

The momentum-weighted track charge, commonly referred to as "jet charge", is defined as:

$$
Q_{\text {diff }}=Q_{b}-Q_{\bar{b}}=-\sum_{\text {tracks }} q_{i} \cdot \operatorname{sgn}\left(\vec{p}_{i} \cdot \hat{T}\right)\left|\left(\vec{p}_{i} \cdot \hat{T}\right)\right|^{\kappa},
$$

where $\vec{p}_{i}$ is the $\mathrm{i}^{\text {th }}$ track momentum, $q_{i}$ its charge and $\hat{T}$ the direction of the event thrust axis. The coefficient $\kappa$ was chosen to be 0.5 in order to maximize the analyzing power of the tag. The quantity $Q_{\text {diff }}$ represents the difference between the momentum-weighted charges in the two hemispheres.

This methods $[9,10]$ uses all the available events, hence it is possible for this analysis to estimate the analyzing power directly from the data (self-calibration). The main input from the $\mathrm{MC}$ is the hemisphere correlation.

The forward-backward asymmetries obtained for left- and right-handed incident electrons are shown in fig 2. A maximum likelihood analysis on the entire data sample yields: $A_{b}=$ $0.882 \pm 0.020$ (stat) \pm 0.029 (syst).

\section{3 $A_{b}$ and $A_{c}$ with a Lepton Tag}

$A_{b}$ and $A_{c}$ can also be measured by tagging bottom and charmed hadrons using their semileptonic decays[11]. The lepton total and transverse momenta (with respect to the nearest jet) are used to calculate the probabilities that a lepton comes from each of the possible physics processes: $Z^{0} \rightarrow b \bar{b}, b \rightarrow l ; Z^{0} \rightarrow b \bar{b}, \bar{b} \rightarrow \bar{c} \rightarrow l ; Z^{0} \rightarrow b \bar{b}, b \rightarrow \bar{c} \rightarrow l ; Z^{0} \rightarrow c \bar{c}, \bar{c} \rightarrow l$; and background (leptons from light hadron decays, photon conversions, and misidentified hadrons). The lepton charge provides quark-antiquark discrimination, while the direction of the jet nearest to the lepton approximates the direction of underlying quark.

Electrons are identified[12] with both calorimeter and CRID information for tracks with

$p>2 \mathrm{GeV} / \mathrm{c}$ in the angular range $|\cos \theta|<0.72$. This information is incorporated into 


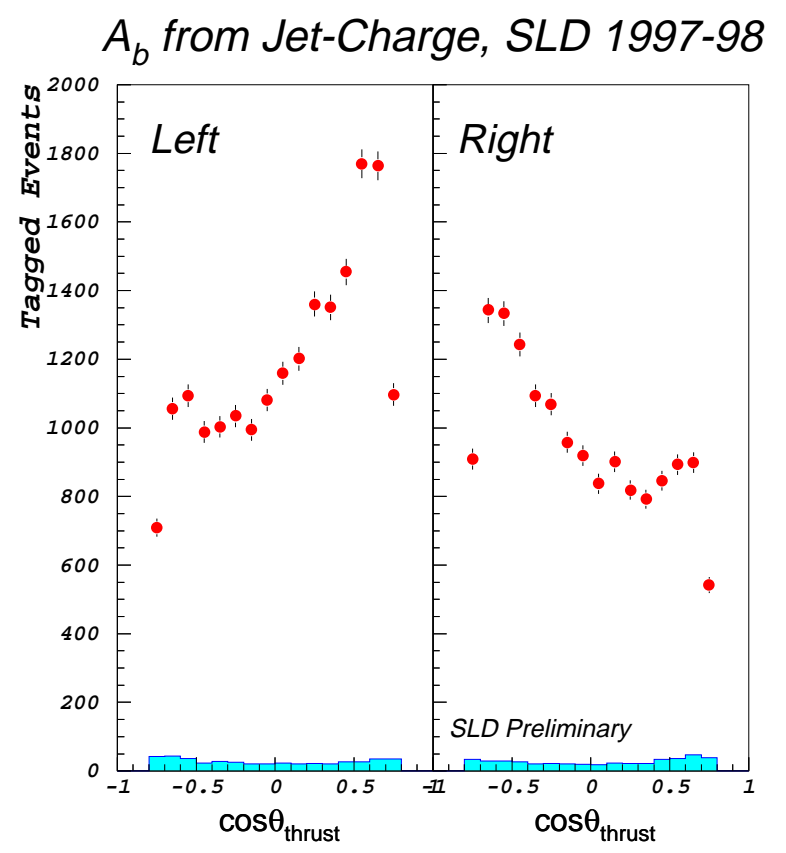

Figure 2: Distributions of the cosine of the thrust axis $\left|\cos \theta_{\text {thrust }}\right|$ for left- and right-handed electron beams for the jet-charge analysis (1997-98 data).

a neural network trained on Monte Carlo electrons. The efficiency (purity) for electron identification is on average $62 \%(70 \%)$ and over $78 \%(80 \%)$ for electrons with momenta greater than $15 \mathrm{GeV} / \mathrm{c}$. Muon identification[13] is performed for tracks with $p>2 \mathrm{GeV} / \mathrm{c}$ in the angular range $|\cos \theta|<0.70$ and uses information from the tracking and the Warm Iron Calorimeter. As for the electron identification, CRID information is added to reject $\pi$, $\mathrm{K}$, and p background. The simulated prompt muon identification efficiency is $81 \%$, with a purity of $68 \%$. Both muon and electron identification algorithms have been tested on control samples from data.

This analysis presents the following new features:

- In case of an identified electron in the event, a secondary vertex is required in either hemisphere to achieve $u d s$ rejection.

- When an identified muon is present, the vertex mass and the $L / D$ variable of the muon tracks (see fig 3) are included in the probability function together with the total and transverse lepton momenta.

As shown in fig $4, L / D$ discriminates between muons from direct and cascade $b$ decays. This is due to the fact that the topological vertexing algorithm often finds only one vertex for both the $B$ and the $D$ decay, thus $L / D<(>) 1$ indicates a muon from direct (cascade) $b$ decay.

A maximum likelihood analysis, applied to all hadronic $Z^{0}$ events containing leptons, is used to determine $A_{b}$ and $A_{c}$ simultaneously. We measure: $A_{b}=0.924 \pm 0.032$ (stat) \pm 0.026 (syst) and $A_{c}=0.567 \pm 0.051$ (stat) \pm 0.064 (syst). 


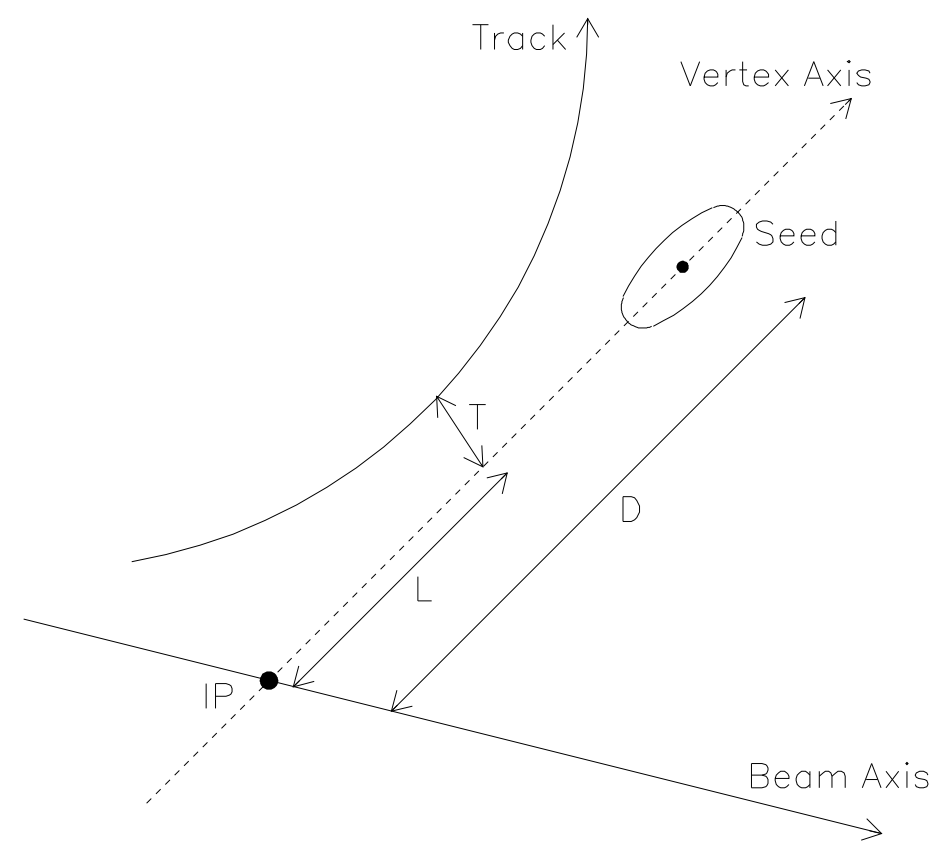

Figure 3: Topological parameters of a track: D is the distance of the secondary vertex (seed) from the interaction point along the line connecting them and $\mathrm{L}$ the distance from the IP of the projection of the point of closest approach on the vertex axis.

\section{4 $A_{c}$ with Exclusive $D$ Mesons}

In this analysis six modes are exclusively reconstructed to tag the charm quark: $D^{*} \rightarrow$ $D^{0} \pi_{\text {soft }}$ with $D^{0}$ decaying into $K \pi, K \pi \pi^{0}, K \pi \pi \pi$ and $K l \nu, D^{+} \rightarrow K^{+} \pi^{+} \pi^{-}$and $D^{0} \rightarrow$ $K^{-} \pi^{+}$. The vertex information is used to reject both $b$ and $u d s$ background. The reconstruction efficiency is low $(\sim 4 \%)$, but the high correct sign probability and the good determination of the underlying charm quark direction assures very low systematic errors.

The charm signal is obtained by selecting a signal region in the invariant mass distribution of the $\mathrm{D}$ meson (see fig 5). For the $D^{*}$ the selection is improved with a cut on the $D^{*}-D^{0}$ mass difference. The background under the signal is estimated from the sidebands.

An unbinned likelihood analysis to the full differential cross section is used to extract: $A_{c}=0.690 \pm 0.042$ (stat) \pm 0.022 (syst).

\section{5 $A_{c}$ with Inclusive Soft Pion}

In this analysis the charm quark is tagged by the presence of a slow pion from the decay $D^{*} \rightarrow D^{0} \pi_{\text {soft }}$. In this decay the pion is produced along the $D^{*}$ jet direction $\left(P_{T}^{2} \sim 0\right)$. Fig 6 shows the distribution of the $P_{T}^{2}$ signal. A signal to background ratio of 1:2 can be achieved with similar vertexing cut as for the previous analysis The result of this method is: $A_{c}=0.683 \pm 0.052$ (stat) \pm 0.050 (syst).

\section{6 $A_{c}$ with Vertex Charge and Identified Kaons}

The same charm tag used in the $R_{c}$ measurement is used in this analysis[14]. Furthermore we required at least one hemisphere passing the charm selection, while neither hemisphere 


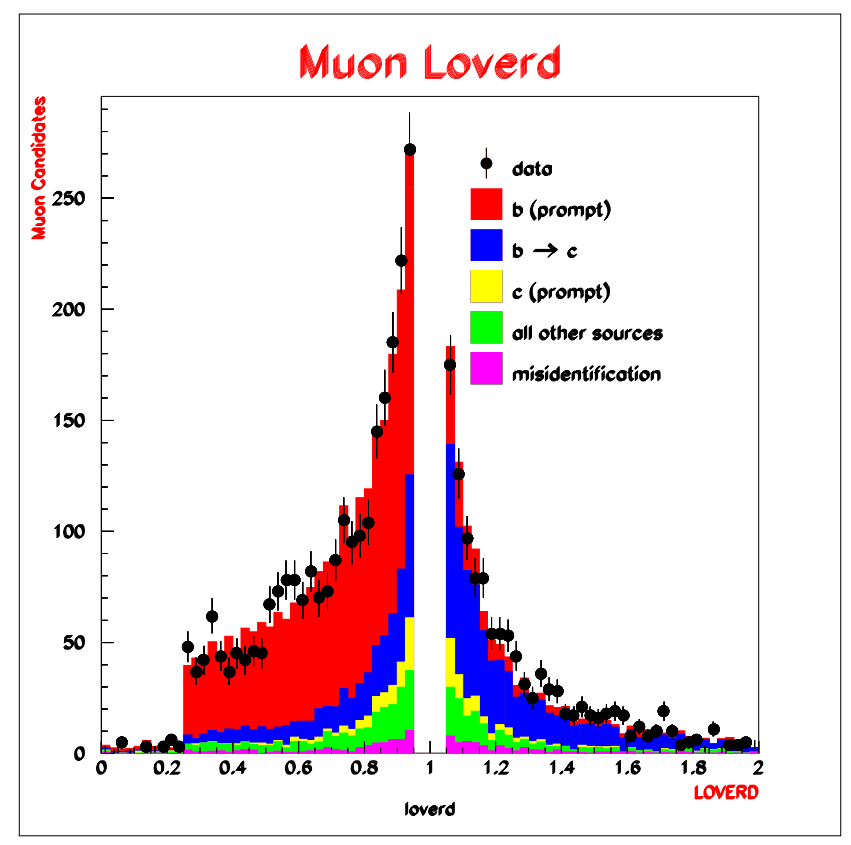

Figure 4: Tails of the L/D distribution for muons in 1996-8 data (dots) and Monte Carlo (histogram).

passing the bottom selection. The final charm efficiency is $28 \%$ with a purity of $82 \%$. The quark charge is given by the charge of an identified kaon in the event (which is present in $\sim 25 \%$ of the selected events and gives $90 \%$ correct sign fraction) or by the vertex charge (present in $\sim 25 \%$ of the events with $91 \%$ correct sign fraction). This gives high statistics, hence the possibility to self-calibrate also this analysis. The results for the 1993-97 data sample is: $A_{c}=0.650 \pm 0.041$ (stat) \pm 0.033 (syst).

\section{$6 \quad A_{s}$ Measurement}

$s \bar{s}$ events at SLD can be effectively identified thanks mainly to the good separation of tracks from secondary vertices and the contribution of the CRID to particle identification. $c \bar{c}$ and $b \bar{b}$ events are suppressed by requiring no tracks inconsistent with the IP. Each thrust hemisphere of a light-flavor tagged event is then required to contain at least one identified strange particle with large momentum $\left(K^{ \pm}\right.$with $p>9 \mathrm{GeV}$ and $K_{s}^{0}$ or $\Lambda^{0} / \bar{\Lambda}^{0}$ with $p>5$ $\mathrm{GeV})$. Strange hemispheres are tagged using the highest momentum strange particle present in the hemisphere. A tag is required in both hemispheres and at least one of the tags must be signed. If both are signed, signs must be opposite.

Using these criteria we obtain an overall $s \bar{s}$ purity of $69 \%$. The initial $s$ quark direction is approximated by the thrust axis of the event, signed to point in the direction of negative strangeness, whose distributions for negative and positive polarization events are shown in fig 7 .

The result, the most precise in the world, is: $A_{s}=0.82 \pm 0.10$ (stat) \pm 0.08 (syst)[15]. 

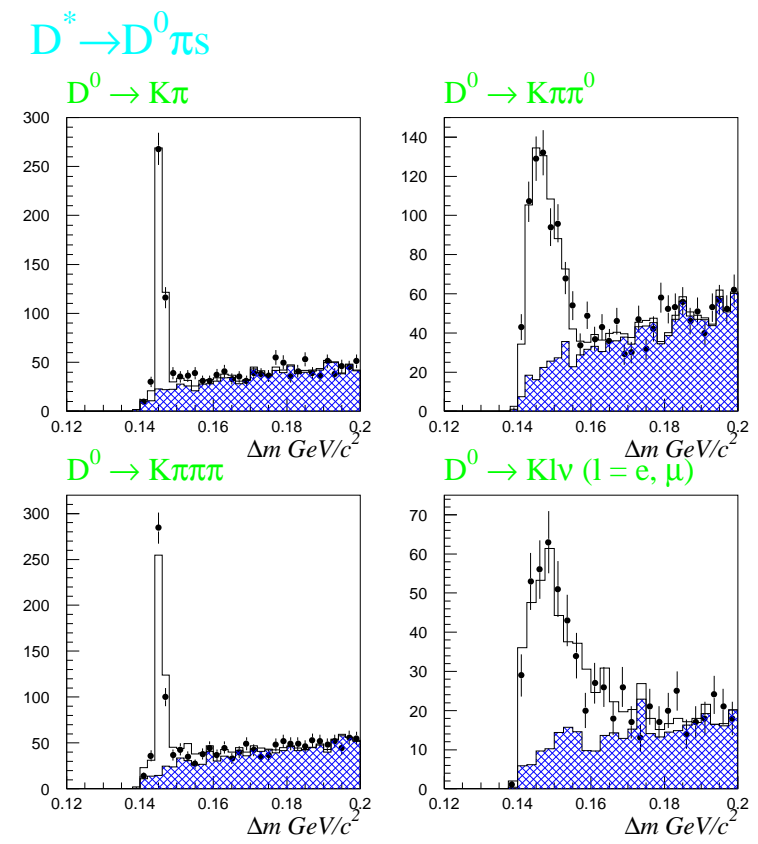

Figure 5: Invariant mass for $D \rightarrow K \pi \pi$ and $D^{0} \rightarrow K \pi$ reconstructed decays.
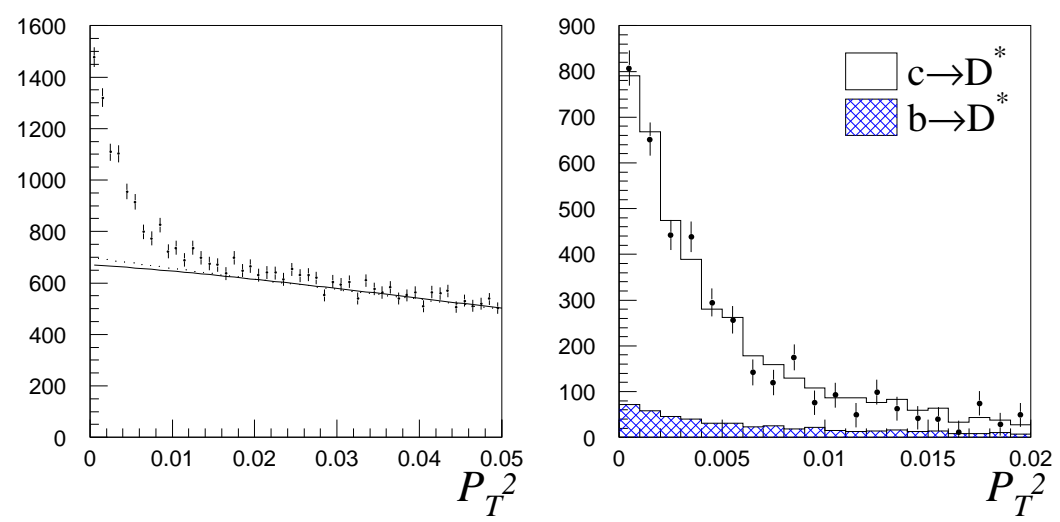

Figure 6: Distribution of the transverse momentum (with respect to the jet axis) of the soft pion candidates. Left: full sample. Right: after background subtraction.

\section{Conclusions}

We have presented the results of several important electroweak measurements performed by the SLD Collaboration. The $A_{L R}$ and $A_{\text {lepton }}$ measurements yield: $\sin ^{2} \theta_{W}=0.23109 \pm$ 0.00029. It is interesting to note that this result is in agreement with the LEP leptonic asymmetry combined result $\left(\sin ^{2} \theta_{W}=0.23153 \pm 0.00034\right)$, but differs by over 2 standard deviations from the LEP results obtained from "hadron-only" measurements $\left(A_{F B}^{b}, A_{F B}^{c}\right.$, and $\left.Q_{F B}\right)$.

Both the $R_{c}$ and the $R_{b}$ results are in good agreement with the Standard Model predictions as well as with the LEP results. The SLD $R_{c}$ measurement constitutes the most precise single measurement of this observable in the world.

The three $A_{b}$ results described previously have been combined taking into account the small statistical and systematic correlations. The resulting SLD average is $A_{b}(\mathrm{SLD})=$ 

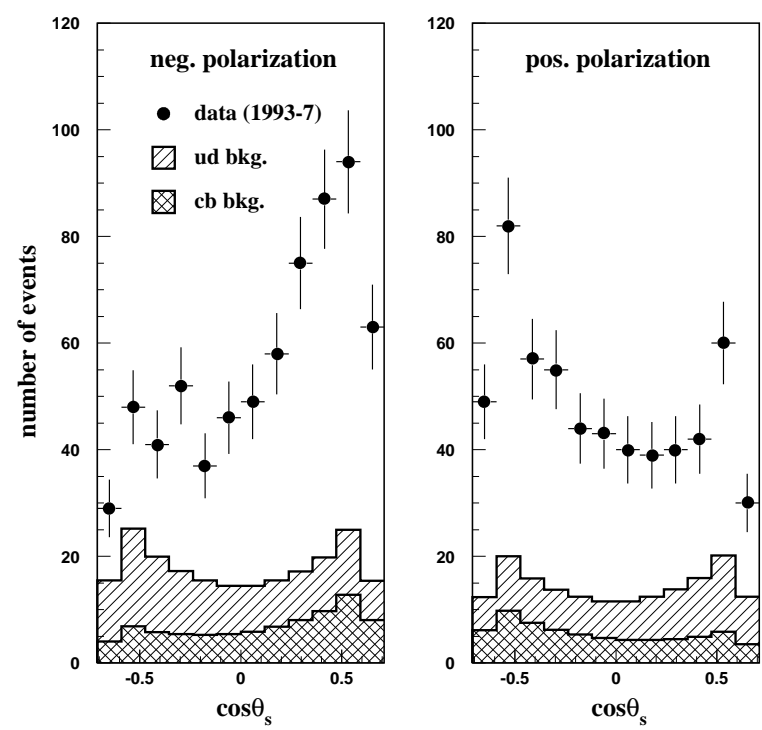

Figure 7: Distributions of the thrust axis for left- and right-handed events tagged as $s \bar{s}$.

$0.898 \pm 0.029$. This value can be compared with the LEP results, which use the unpolarized forward-backward asymmetry for $b$ quarks $\left(A_{F B}^{b}(\mathrm{LEP})=0.0991 \pm 0.0020\right)$. Since the simple forward-backward asymmetry does not measure directly $A_{b}$, we need to use the world average value for $A_{e}=0.1489 \pm 0.0017$, derived combining the SLD $A_{L R}$ and the LEP $A_{l}$ measurements. The derived LEP average is then: $A_{b}(\mathrm{LEP})=\frac{4}{3} \frac{A_{F B}^{b}}{A_{e}}=0.887 \pm 0.021$ for a combined SLD and LEP average of $A_{b}=0.891 \pm 0.017$, which is about 2.6 standard deviations below the Standard Model prediction of 0.935 .

Similar considerations are valid for the $A_{c}$ measurements. The SLD average result is: $A_{c}(\mathrm{SLD})=0.663 \pm 0.032$, which is in agreeement with both the derived LEP average $A_{c}(\mathrm{LEP})=0.635 \pm 0.040$ and the Standard Model Prediction of 0.667.

\section{Acknowledgements}

I would like to thank all the SLD collaborators for their support and efforts and the SLAC accelerator department for its outstanding performance. I'd like to thank especially Nicolo de Groot and Sal Fahey for their help in writing this manuscript. And, finally, I'm grateful to the organizators of Les Recontres de Physique de la Vallee d'Aoste for inviting me and for the excellent hospitality. This work was supported in part by DOE Contract DE-AC0376SF00515(SLAC).

\section{References}

[1] The LEP collaborations and the LEP electroweak working group, CERN-EP/99-15 and CERN-PPE/97-154 and references therein.

[2] K. Abe et al, Phys. Rev. D 53, 1023 (1996). 
Table 1: Summary of the results of SLD electroweak measurements

\begin{tabular}{|c|l|}
\hline Observable & Prelim. Result \\
\hline \hline$A_{L R}$ & $0.1510 \pm 0.0025$ \\
\hline $\sin ^{2} \theta_{W}$ (from $\left.A_{L R}\right)$ & $0.23101 \pm 0.00031$ \\
\hline$A_{e}\left(\right.$ from $\left.\tilde{A}_{F B}\right)$ & $0.1504 \pm 0.0072$ \\
$A_{\mu}$ & $0.120 \pm 0.019$ \\
$A_{\tau}$ & $0.142 \pm 0.019$ \\
\hline $\sin ^{2} \theta_{W}$ (from $A_{L R}$ and $\left.A_{l}\right)$ & $0.23109 \pm 0.00029$ \\
\hline$A_{c}$ - vertex charge+kaons & $0.650 \pm 0.041 \pm 0.033$ \\
$A_{c}$ - inclusive soft pion & $0.683 \pm 0.052 \pm 0.050$ \\
$A_{c}$ - exclusive D & $0.690 \pm 0.042 \pm 0.022$ \\
$A_{c}$ - lepton tag & $0.567 \pm 0.051 \pm 0.064$ \\
\hline$A_{c}$ - SLD average & $0.663 \pm 0.032$ \\
\hline$A_{b}$ - jet charge & $0.882 \pm 0.020 \pm 0.029$ \\
$A_{b}$ - kaon tag & $0.855 \pm 0.088 \pm 0.102$ \\
$A_{b}$ - lepton tag & $0.924 \pm 0.032 \pm 0.026$ \\
\hline$A_{b}$ - SLD average & $0.898 \pm 0.029$ \\
\hline$A_{s}$ & $0.82 \pm 0.10 \pm 0.08$ \\
\hline$R_{c}$ & $0.1685 \pm 0.0047 \pm 0.0043$ \\
$R_{b}$ & $0.2159 \pm 0.0014 \pm 0.0014$ \\
\hline
\end{tabular}

[3] K. Abe et al, SLAC-PUB-7878, Contributed to ICHEP 1998.

[4] K. Abe et al, Phys. Rev. Lett. 79, 804 (1997).

[5] K. Abe et al, Phys. Rev. Lett. 80, 660 (1998).

[6] K. Abe et al, SLAC-PUB-7880, Contributed to ICHEP 1998.

[7] D. Jackson, Nucl. Instrum. Methods A338, 247 (1997).

[8] K. Abe et al., SLAC-PUB-7959, to be published in Phys. Rev. Lett.

[9] K. Abe et al, SLAC-PUB-7886, Contributed to ICHEP 1998.

[10] K. Abe et al, Phys. Rev. Lett. 81, 942 (1998).

[11] K. Abe et al, SLAC-PUB-7798, submitted to Phys. Rev. Lett.

[12] D. Falciai, Ph.D. Thesis, Universitá di Perugia (1996).

[13] G. Mancinelli, Ph.D. Thesis, Universitá di Perugia (1997).

[14] K. Abe et al, SLAC-PUB-7879, Contributed to ICHEP 1998.

[15] K. Abe et al, SLAC-PUB-7825, Contributed to ICHEP 1998. 


\section{${ }^{\dagger}$ The SLD Collaboration}

Kenji Abe, ${ }^{(20)}$ Koya Abe, ${ }^{(32)}$ T. Abe, ${ }^{(28)}$ I.Adam, ${ }^{(28)}$ T. Akagi, ${ }^{(28)}$ N. J. Allen, ${ }^{(4)}$

A. Arodzero, ${ }^{(21)}$ W.W. Ash, ${ }^{(28)}{ }^{*}$ D. Aston, ${ }^{(28)}$ K.G. Baird,${ }^{(16)}$ C. Baltay, ${ }^{(38)}$ H.R. Band, ${ }^{(37)}$

M.B. Barakat, ${ }^{(15)}$ O. Bardon, ${ }^{(18)}$ T.L. Barklow, ${ }^{(28)}$ G. L. Bashindzhagyan, ${ }^{(19)}$

J.M. Bauer, ${ }^{(17)}$ G. Bellodi, ${ }^{(22)}$ R. Ben-David, ${ }^{(38)}$ A.C. Benvenuti, ${ }^{(3)}$ G.M. Bilei, ${ }^{(24)}$

D. Bisello, ${ }^{(23)}$ G. Blaylock, ${ }^{(16)}$ J.R. Bogart, ${ }^{(28)}$ B. Bolen, ${ }^{(17)}$ G.R. Bower, ${ }^{(28)}$ J. E. Brau, ${ }^{(21)}$ M. Breidenbach, ${ }^{(28)}$ W.M. Bugg, ${ }^{(31)}$ D. Burke, ${ }^{(28)}$ T.H. Burnett, ${ }^{(36)}$ P.N. Burrows, ${ }^{(22)}$ R.M. Byrne, ${ }^{(18)}$ A. Calcaterra, ${ }^{(11)}$ D. Calloway, ${ }^{(28)}$ B. Camanzi, ${ }^{(10)}$ M. Carpinelli, ${ }^{(25)}$

R. Cassell, ${ }^{(28)}$ R. Castaldi, ${ }^{(25)}$ A. Castro, ${ }^{(23)}$ M. Cavalli-Sforza, ${ }^{(34)}$ A. Chou, ${ }^{(28)}$ E. Church, ${ }^{(36)}$ H.O. Cohn, ${ }^{(31)}$ J.A. Coller, ${ }^{(5)}$ M.R. Convery, ${ }^{(28)}$ V. Cook, ${ }^{(36)}$ R. Cotton, ${ }^{(4)}$ R.F. Cowan, ${ }^{(18)}$ D.G. Coyne, ${ }^{(34)}$ G. Crawford, ${ }^{(28)}$ C.J.S. Damerell, ${ }^{(26)}$ M. N. Danielson, ${ }^{(7)}$ M. Daoudi, ${ }^{(28)}$ N. de Groot, ${ }^{(28)}$ R. Dell'Orso, ${ }^{(24)}$ P.J. Dervan, ${ }^{(4)}$ R. de Sangro, ${ }^{(11)}$

M. Dima, ${ }^{(9)}$ A. D'Oliveira, ${ }^{(6)}$ D.N. Dong, ${ }^{(18)}$ M. Doser, ${ }^{(28)}$ R. Dubois ${ }^{(28)}$ B.I. Eisenstein, ${ }^{(12)}$ V. Eschenburg, ${ }^{(17)}$ E. Etzion, ${ }^{(37)}$ S. Fahey, ${ }^{(7)}$ D. Falciai, ${ }^{(11)}$ C. Fan, ${ }^{(7)}$ J.P. Fernandez, ${ }^{(34)}$ M.J. Fero, ${ }^{(18)}$ K.Flood, ${ }^{(16)}$ R. Frey, ${ }^{(21)}$ T. Gillman, ${ }^{(26)}$ G. Gladding, ${ }^{(12)}$ S. Gonzalez, ${ }^{(18)}$

E. R. Goodman, ${ }^{(7)}$ E.L. Hart, ${ }^{(31)}$ J.L. Harton, ${ }^{(9)}$ A. Hasan, ${ }^{(4)}$ K. Hasuko, ${ }^{(32)}$

S. J. Hedges, ${ }^{(5)}$ S.S. Hertzbach, ${ }^{(16)}$ M.D. Hildreth, ${ }^{(28)}$ J. Huber, ${ }^{(21)}$ M.E. Huffer, ${ }^{(28)}$ E.W. Hughes, ${ }^{(28)}$ X.Huynh, ${ }^{(28)}$ H. Hwang, ${ }^{(21)}$ M. Iwasaki, ${ }^{(21)}$ D. J. Jackson, ${ }^{(26)}$ P. Jacques, ${ }^{(27)}$ J.A. Jaros, ${ }^{(28)}$ Z.Y. Jiang, ${ }^{(28)}$ A.S. Johnson, ${ }^{(28)}$ J.R. Johnson, ${ }^{(37)}$ R.A. Johnson, ${ }^{(6)}$ T. Junk, ${ }^{(28)}$ R. Kajikawa, ${ }^{(20)}$ M. Kalelkar, ${ }^{(27)}$ Y. Kamyshkov, ${ }^{(31)}$ H.J. Kang, ${ }^{(27)}$ I. Karliner, ${ }^{(12)}$ H. Kawahara, ${ }^{(28)}$ Y. D. Kim, ${ }^{(29)}$ R. King, ${ }^{(28)}$ M.E. King, ${ }^{(28)}$

R.R. Kofler, ${ }^{(16)}$ N.M. Krishna, ${ }^{(7)}$ R.S. Kroeger, ${ }^{(17)}$ M. Langston, ${ }^{(21)}$ A. Lath, ${ }^{(18)}$

D.W.G. Leith, ${ }^{(28)}$ V. Lia, ${ }^{(18)}$ C.-J. S. Lin, ${ }^{(16)}$ X. Liu, ${ }^{(34)}$ M.X. Liu, ${ }^{(38)}$ M. Loreti, ${ }^{(23)}$

A. Lu, ${ }^{(33)}$ H.L. Lynch, ${ }^{(28)}$ J. Ma, ${ }^{(36)}$ M. Mahjouri, ${ }^{(18)}$ G. Mancinelli, ${ }^{(27)}$ S. Manly, ${ }^{(38)}$

G. Mantovani, ${ }^{(24)}$ T.W. Markiewicz, ${ }^{(28)}$ T. Maruyama, ${ }^{(28)}$ H. Masuda, ${ }^{(28)}$ E. Mazzucato, ${ }^{(10)}$

A.K. McKemey, ${ }^{(4)}$ B.T. Meadows,${ }^{(6)}$ G. Menegatti, ${ }^{(10)}$ R. Messner, ${ }^{(28)}$ P.M. Mockett, ${ }^{(36)}$

K.C. Moffeit, ${ }^{(28)}$ T.B. Moore, ${ }^{(38)}$ M.Morii, ${ }^{(28)}$ D. Muller, ${ }^{(28)}$ V.Murzin, ${ }^{(19)}$ T. Nagamine, ${ }^{(32)}$ S. Narita, ${ }^{(32)}$ U. Nauenberg, ${ }^{(7)}$ H. Neal, ${ }^{(28)}$ M. Nussbaum, ${ }^{(6), *}$ N.Oishi, ${ }^{(20)}$

D. Onoprienko, ${ }^{(31)}$ L.S. Osborne, ${ }^{(18)}$ R.S. Panvini, ${ }^{(35)}$ H. Park, ${ }^{(21)}$ C. H. Park, ${ }^{(30)}$

T.J. Pavel, ${ }^{(28)}$ I. Peruzzi, ${ }^{(11)}$ M. Piccolo, ${ }^{(11)}$ L. Piemontese, ${ }^{(10)}$ E. Pieroni, ${ }^{(25)}$ K.T. Pitts, ${ }^{(21)}$

R.J. Plano, ${ }^{(27)}$ R. Prepost, ${ }^{(37)}$ C.Y. Prescott, ${ }^{(28)}$ G.D. Punkar, ${ }^{(28)}$ J. Quigley, ${ }^{(18)}$

B.N. Ratcliff, ${ }^{(28)}$ T.W. Reeves, ${ }^{(35)}$ J. Reidy, ${ }^{(17)}$ P.L. Reinertsen, ${ }^{(34)}$ P.E. Rensing, ${ }^{(28)}$

L.S. Rochester, ${ }^{(28)}$ P.C. Rowson, ${ }^{(8)}$ J.J. Russell, ${ }^{(28)}$ O.H. Saxton, ${ }^{(28)}$ T. Schalk, ${ }^{(34)}$

R.H. Schindler, ${ }^{(28)}$ B.A. Schumm, ${ }^{(34)}$ J. Schwiening, ${ }^{(28)}$ S. Sen, ${ }^{(38)}$ V.V. Serbo, ${ }^{(28)}$

M.H. Shaevitz, ${ }^{(8)}$ J.T. Shank, ${ }^{(5)}$ G. Shapiro, ${ }^{(14)}$ D.J. Sherden, ${ }^{(28)}$ K. D. Shmakov, ${ }^{(31)}$

C. Simopoulos, ${ }^{(28)}$ N.B. Sinev, ${ }^{(21)}$ S.R. Smith, ${ }^{(28)}$ M. B. Smy ${ }^{(9)}$ J.A. Snyder, ${ }^{(38)}$ H. Staengle, ${ }^{(9)}$ A. Stahl, ${ }^{(28)}$ P. Stamer, ${ }^{(27)}$ R. Steiner, ${ }^{(1)}$ H. Steiner, ${ }^{(14)}$ M.G. Strauss, ${ }^{(16)}$

D. Su, ${ }^{(28)}$ F. Suekane, ${ }^{(32)}$ A. Sugiyama, ${ }^{(20)}$ S. Suzuki, ${ }^{(20)}$ M. Swartz, ${ }^{(13)}$ A. Szumilo, ${ }^{(36)}$

T. Takahashi, ${ }^{(28)}$ F.E. Taylor, ${ }^{(18)}$ J. Thom, ${ }^{(28)}$ E. Torrence, ${ }^{(18)}$ N. K. Toumbas, ${ }^{(28)}$

T. Usher, ${ }^{(28)}$ C. Vannini, ${ }^{(25)}$ J. Va'vra, ${ }^{(28)}$ E. Vella, ${ }^{(28)}$ J.P. Venuti, ${ }^{(35)}$ R. Verdier ${ }^{(18)}$

P.G. Verdini, ${ }^{(25)}$ S.R. Wagner, ${ }^{(28)}$ D. L. Wagner, ${ }^{(7)}$ A.P. Waite,${ }^{(28)}$ S. Walston, ${ }^{(21)}$

J.Wang, ${ }^{(28)}$ C. Ward, ${ }^{(4)}$ S.J. Watts, ${ }^{(4)}$ A.W. Weidemann, ${ }^{(31)}$ E. R. Weiss, ${ }^{(36)}$

J.S. Whitaker, ${ }^{(5)}$ S.L. White, ${ }^{(31)}$ F.J. Wickens, ${ }^{(26)}$ B. Williams, ${ }^{(7)}$ D.C. Williams, ${ }^{(18)}$

S.H. Williams, ${ }^{(28)}$ S. Willocq, ${ }^{(28)}$ R.J. Wilson, ${ }^{(9)}$ W.J. Wisniewski, ${ }^{(28)}$ J. L. Wittlin, ${ }^{(16)}$

M. Woods, ${ }^{(28)}$ G.B. Word, ${ }^{(35)}$ T.R. Wright, ${ }^{(37)}$ J. Wyss, ${ }^{(23)}$ R.K. Yamamoto, ${ }^{(18)}$

J.M. Yamartino, ${ }^{(18)}$ X. Yang, ${ }^{(21)}$ J. Yashima, ${ }^{(32)}$ S.J. Yellin, ${ }^{(33)}$ C.C. Young, ${ }^{(28)}$ H. Yuta, ${ }^{(2)}$ G. Zapalac, ${ }^{(37)}$ R.W. Zdarko, ${ }^{(28)}$ J. Zhou. ${ }^{(21)}$

(The SLD Collaboration) 
(1) Adelphi University, Garden City, New York 11530,

${ }^{(2)}$ Aomori University, Aomori, 030 Japan,

(3) INFN Sezione di Bologna, I-40126, Bologna Italy,

${ }^{(4)}$ Brunel University, Uxbridge, Middlesex, UB8 3PH United Kingdom,

(5) Boston University, Boston, Massachusetts 02215,

(6) University of Cincinnati, Cincinnati, Ohio 45221,

(7) University of Colorado, Boulder, Colorado 80309,

${ }^{(8)}$ Columbia University, New York, New York 10533,

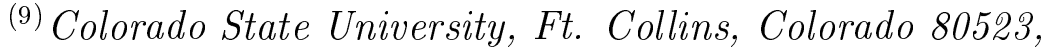

${ }^{(10)}$ INFN Sezione di Ferrara and Universita di Ferrara, I-44100 Ferrara, Italy,

(11) INFN Lab. Nazionali di Frascati, I-00044 Frascati, Italy,

(12) University of Illinois, Urbana, Illinois 61801,

(13) Johns Hopkins University, Baltimore, Maryland 21153,

${ }^{(14)}$ Lawrence Berkeley Laboratory, University of California, Berkeley, California 94720,

${ }^{(15)}$ Louisiana Technical University - Ruston, LA 71272,

(16) University of Massachusetts, Amherst, Massachusetts 01003,

(17) University of Mississippi, University, Mississippi 3867\%,

${ }^{(18)}$ Massachusetts Institute of Technology, Cambridge, Massachusetts 02139,

${ }^{(19)}$ Institute of Nuclear Physics, Moscow State University, 119899, Moscow Russia,

${ }^{(20)}$ Nagoya University, Chikusa-ku, Nagoya 464 Japan,

(21) University of Oregon, Eugene, Oregon 97403,

${ }^{(22)}$ Oxford University, Oxford, OX1 3RH, United Kingdom,

${ }^{(23)}$ INFN Sezione di Padova and Universita di Padova I-35100, Padova, Italy,

${ }^{(24)}$ INFN Sezione di Perugia and Universita di Perugia, I-06100 Perugia, Italy,

${ }^{(25)}$ INFN Sezione di Pisa and Universita di Pisa, I-56010 Pisa, Italy,

${ }^{(26)}$ Rutherford Appleton Laboratory, Chilton, Didcot, Oxon OX11 0QX United Kingdom,

${ }^{(27)}$ Rutgers University, Piscataway, New Jersey 08855,

${ }^{(28)}$ Stanford Linear Accelerator Center, Stanford University, Stanford, California 94309,

${ }^{(29)}$ Sogang University, Seoul, Korea,

${ }^{(30)}$ Soongsil University, Seoul, Korea 156-743,

(31) University of Tennessee, Knoxville, Tennessee 37996 ,

(32) Tohoku University, Sendai 980, Japan,

(33) University of California at Santa Barbara, Santa Barbara, California 93106,

(34) University of California at Santa Cruz, Santa Cruz, California 95064,

${ }^{(35)}$ Vanderbilt University, Nashville, Tennessee 37235,

${ }^{(36)}$ University of Washington, Seattle, Washington 98105 ,

(37) University of Wisconsin, Madison, Wisconsin 53706 ,

(38) Yale University, New Haven, Connecticut 06511.

* Deceased. 